\title{
Controllability of Linear Discrete-Time Systems with Both Delayed States and Delayed Inputs
}

\author{
Hong Shi, ${ }^{1}$ Guangming Xie, ${ }^{2,3}$ and Wenguang Luo ${ }^{4}$ \\ ${ }^{1}$ Department of Mathematics and Physics, Beijing Institute of Petrochemical Technology, Beijing 102617, China \\ ${ }^{2}$ State Key Laboratory for Turbulence and Complex Systems, College of Engineering, Peking University, Beijing 100871, China \\ ${ }^{3}$ School of Electrical and Electronics Engineering, East China Jiaotong University, Nanchang 330013, China \\ ${ }^{4}$ School of Electric and Information Engineering, Guangxi University of Science and Technology, Liuzhou 545006, China
}

Correspondence should be addressed to Guangming Xie; xiegming@pku.edu.cn

Received 29 November 2012; Accepted 31 January 2013

Academic Editor: Valery Y. Glizer

Copyright (c) 2013 Hong Shi et al. This is an open access article distributed under the Creative Commons Attribution License, which permits unrestricted use, distribution, and reproduction in any medium, provided the original work is properly cited.

The controllability issues for discrete-time linear systems with delay in state and control are addressed. By introducing a new concept, the controllability realization index (CRI), the characteristic of controllability is revealed. An easily testable necessary and sufficient condition for the controllability of discrete-time linear systems with state and control delay is established.

\section{Introduction}

The concept of controllability, first given by Kalman in the 1960s [1], plays a fundamental role in the modern control theory and has close connections with pole assignment, structure decomposition, quadratic control, and so forth $[2,3]$. The various aspects of the controllability of linear systems with delay were considered by several authors [4-11]. The discrete cases have been considered by Klamka [7], Watanabe [8], and Phat [9], but the mathematical conditions given for investigating the controllability are not suitable for real verification and application.

In our recent paper [12], a new concept called controllability realization index (CRI) is proposed, which is crucial in determining the controllability of such kind of discrete systems with delays. In that paper, it is proved that the value of CRI is finite for discrete systems with delays, and a general CRI value for planar discrete systems with delays is given. Thus, the judging condition of controllability for planar case is established. In this paper, we will extend our result to the more general case, namely, discrete systems with any order, with time delays both in state and in control.

This paper is organized as follows. In Section 2, some basic definitions and preliminary results are presented. Section 3 is the main results. An easily testable necessary and sufficient condition for the controllability of discretetime linear systems with state and control delay is established. A numerical example is given in Section 4. Finally, the conclusion is provided in Section 5 .

\section{Problem Formulation and Preliminaries}

In this paper, we consider the discrete-time case the system model is described as follows:

$$
x(k+1)=\sum_{i=0}^{p} A_{i} x(k-i)+\sum_{j=0}^{q} B_{j} u(k-j),
$$

where $x(k) \in \mathbb{R}^{n}$ is the state, $u(k) \in \mathbb{R}^{s}$ is the input, $A_{i} \in \mathbb{R}^{n \times n}$ and $B_{j} \in \mathbb{R}^{n \times s}$ are constant matrices, and the positive integers $i, j$ are the lengths of the steps of time delays. The initial states $x(-p), x(-p+1), \ldots, x(0)$ and the initial input $u(-q), u(-q+$ $1), \ldots, u(-1)$ are given arbitrarily.

The controllability discussed here refers to the unconstrained controllability or completely controllability.

Definition 1 (controllability). The system (1) is said to be (completely) controllable if, for any initial input $u(-q), u(-q+$ $1), \ldots, u(-1)$, any initial state $x(-p), x(-p+1), \ldots, x(0)$, and 
any terminal state $x_{f}$, there exist a positive integer $k$ and, input $u(0), \ldots, u(k-1)$ such that $x(k)=x_{f}$.

Definition 2 (controllability realization index, CRI). For the system (1), if there exists a positive integer $K$ such that for any initial input $u(-q), u(-q+1), \ldots, u(-1)$, initial state $x(-p), x(-p+1), \ldots, x(0)$, and any terminal state $x_{f}$, there exists an input $u(0), \ldots, u(K-1)$ such that $x(K)=x_{f}$, then one calls $K$ the controllability realization index (CRI) of the system (1). Obviously, if exists, such $K$ is not unique, so the one calls the smallest $K$ among them the minimum controllability realization index (MinCRI).

Denote by $\mathbb{N}$ the nonnegative integer set. The matrices $A_{1}, \ldots, A_{N} \in \mathbb{R}^{n \times n}$ are said to be linearly dependent on $\mathbb{R}^{n \times n}$, if there exist scalars $c_{1}, \ldots, c_{N} \in \mathbb{R}$, not all are zero, such that $\sum_{i=1}^{N} c_{i} A_{i}=0$. In the following statement, $\operatorname{span}\left\{A_{1}, \ldots, A_{N}\right\}$ will be used to denote the space constructed by the linear combinations of matrices $\left\{A_{1}, \ldots, A_{N}\right\}$.

\section{Main Results}

3.1. Delay in State. In this section, we first investigate the controllability of the systems only with delay in state

$$
x(k+1)=\sum_{i=0}^{p} A_{i} x(k-i)+B u(k)
$$

Now, we introduce a matrix sequence $\left\{G_{k}\right\}_{k=0}^{\infty} \subseteq \mathbb{R}^{n \times n}$ as follows:

$$
G_{k}= \begin{cases}I & \text { if } k=0 \\ \sum_{i=0}^{k-1} A_{i} G_{k-1-i} & \text { if } k=1, \ldots, p \\ \sum_{i=0}^{p} A_{i} G_{k-1-i} & \text { if } k=p+1, p+2, \ldots\end{cases}
$$

Lemma 3. The general solution of the system (2) is given by

$x(k+1)=\Psi(k, x(-p), \ldots, x(0))+\sum_{i=0}^{k} G_{k-i} B u(i), \quad k \in \mathbb{N}$,

where $\Psi(k, x(-p), \ldots, x(0))$ is the part of the solution with zero input.

Proof. See Appendix A.

Lemma 4. The matrix sequence $\left\{G_{k}\right\}_{k=0}^{\infty}$ given by (3) satisfies

$$
\operatorname{span}\left\{G_{0}, \ldots, G_{k}, \ldots\right\}=\operatorname{span}\left\{G_{0}, \ldots, G_{n(p+1)-1}\right\} .
$$

Proof. See Appendix B.

Theorem 5. The system (2) is controllable if and only if $\operatorname{rank}\left[G_{0} B|\cdots| G_{n(p+1)-1} B\right]=n$.
Proof. (Necessity) If the system is controllable, then we know that span $\left\{G_{0}, \ldots, G_{k}, \ldots\right\}=\mathbb{R}^{n}$. Thus, by Lemma 4 , we have $\operatorname{rank}\left[G_{0} B|\cdots| G_{n(p+1)-1} B\right]=n$.

(Sufficiency) By Lemma 3, we have

$$
\begin{aligned}
x(n(p+1))= & \Psi(n(p+1)-1, x(-p), \ldots, x(0)) \\
& +\sum_{i=0}^{n(p+1)-1} G_{n(p+1)-1-i} B u(i) .
\end{aligned}
$$

Since rank $\left[G_{0} B|\cdots| G_{n(p+1)-1} B\right]=n$, for any initial state $x(-p), \ldots, x(0)$ and any terminal state $x(n(p+1))$, we can select appropriate inputs $u(0), \ldots, u(n(p+1)-1)$ such that the equation $x(n(p+1))=x_{f}$, where $x(n(p+1))$ is given by the above equation and $x_{f}$ is arbitrary. Thus, the system is controllable.

Corollary 6. $n(p+1)$ is a CRI of the system (2).

Proof. It is directly followed from Theorem 5.

Remark 7. This work has improved the result in [12]. When the system is second order, that is, $n=2$, we prove $2 p+2$ to be a CRI value, which differs from the CRI value $2 p+4$ in [12]. The difference lies in that the CRI of a system is not unique. For practical applications, obviously the less, the better.

3.2. Delays in Both State and Input. Now we investigate the controllability of the system (1). We only consider the case when $p=q$, for the case $p \neq q$, the discussion is similar (Without loss of generality, we assume that $p>q$, and let $B_{j}=0, j=q+1, q+2, \ldots, p$, then we come back to the $p=q$ case.).

Lemma 8. The solution of the system (1) can be expressed as

$$
\begin{aligned}
x(k+1)= & \Phi(k, x(-p), \ldots, x(0), u(-p), \ldots, u(-1)) \\
& +\sum_{i=0}^{k} H_{k-i} u(i), \quad k \in \mathbb{N},
\end{aligned}
$$

where

$$
H_{k}= \begin{cases}G_{0} B_{0} & \text { if } k=0 \\ \sum_{i=0}^{k} G_{k-i} B_{i} & \text { if } k=1, \ldots, p-1 \\ \sum_{i=0}^{p} G_{k-i} B_{i} & \text { if } k=p, p+1, \ldots ;\end{cases}
$$

and $\Phi(k, x(-p), \ldots, x(0), u(-p), \ldots, u(-1))$ is the part of the solution corresponding only to the initial state and initial input.

Proof. The proof is similar to that of Lemma 3.

Lemma 9. The matrix sequence $\left\{H_{k}\right\}_{k=0}^{\infty}$ given by (8) satisfies

$$
\operatorname{span}\left\{H_{0}, \ldots, H_{k}, \ldots\right\}=\operatorname{span}\left\{H_{0}, \ldots, H_{n(p+1)+p-1}\right\} .
$$


Proof. See Appendix C.

Theorem 10. The system (1) is controllable if and only if rank $\left[H_{0}|\cdots| H_{n(p+1)+p-1}\right]=n$.

Proof. The proof is similar to that of Theorem 5.

Corollary 11. $n(p+1)+p$ is a CRI of the system (1).

Proof. It is directly followed from Theorem 10.

Remark 12. This corollary provides a complete and verifiable method to testify the controllability of a general discretetime system with delay in state or in control or both. Approximately the computation work for each $H_{k}$ is $O\left(p n^{3}\right)$, and the entire testing work takes $O\left(p^{2} n^{4}\right)$.

\section{Example}

In this section, we present a numeric example.

Example 13. Consider the system (1) with $p=q=2, n=$ 3 and

$$
\begin{gathered}
A_{0}=\left[\begin{array}{lll}
1 & 0 & 0 \\
0 & 0 & 0 \\
0 & 0 & 0
\end{array}\right], \quad A_{1}=\left[\begin{array}{lll}
0 & 0 & 0 \\
0 & 1 & 0 \\
0 & 0 & 1
\end{array}\right], \quad A_{2}=\left[\begin{array}{lll}
1 & 0 & 0 \\
0 & 1 & 1 \\
0 & 0 & 1
\end{array}\right], \\
B_{0}=\left[\begin{array}{l}
1 \\
0 \\
0
\end{array}\right], \quad B_{1}=\left[\begin{array}{l}
0 \\
1 \\
0
\end{array}\right], \quad B_{2}=\left[\begin{array}{l}
0 \\
0 \\
1
\end{array}\right] .
\end{gathered}
$$

By simple calculation, we get

$$
\begin{gathered}
G_{0}=\left[\begin{array}{lll}
1 & 0 & 0 \\
0 & 1 & 0 \\
0 & 0 & 1
\end{array}\right], \quad G_{1}=\left[\begin{array}{lll}
1 & 0 & 0 \\
0 & 0 & 0 \\
0 & 0 & 0
\end{array}\right], \quad G_{2}=\left[\begin{array}{lll}
1 & 0 & 0 \\
0 & 1 & 0 \\
0 & 0 & 1
\end{array}\right], \\
G_{3}=\left[\begin{array}{lll}
2 & 0 & 0 \\
0 & 1 & 1 \\
0 & 0 & 1
\end{array}\right], \quad G_{4}=\left[\begin{array}{lll}
3 & 0 & 0 \\
0 & 1 & 0 \\
0 & 0 & 1
\end{array}\right], \\
H_{0}=\left[\begin{array}{l}
1 \\
0 \\
0
\end{array}\right], \quad H_{1}=\left[\begin{array}{l}
1 \\
0 \\
0
\end{array}\right], \quad H_{2}=\left[\begin{array}{l}
1 \\
0 \\
0
\end{array}\right], \\
H_{3}=\left[\begin{array}{l}
2 \\
1 \\
0
\end{array}\right], \quad H_{4}=\left[\begin{array}{l}
4 \\
1 \\
1
\end{array}\right] .
\end{gathered}
$$

Thus, by Theorem 10, the system is controllable.

\section{Conclusion}

In this paper, we have investigated the controllability of discrete-time linear systems with time delays. Necessary and sufficient conditions have been established for discrete-time linear systems with state delay or both state and control delays. The proposed conditions are suitable for real verification and can be efficiently computed.

\section{Appendices}

\section{A. Proof of Lemma 3}

Proof. Now, we prove that (4) holds.

For $k=0$, we have

$$
\begin{aligned}
x(1) & =\sum_{i=0}^{p} A_{i} x(-i)+B u(0) \\
& =\Psi(0, x(-p), \ldots, x(0))+G_{0} B u(0) .
\end{aligned}
$$

For $k=1$, we have

$$
\begin{aligned}
x(2)= & \sum_{i=0}^{p} A_{i} x(1-i)+B u(1) \\
= & \sum_{i=1}^{p} A_{i} x(1-i)+A_{0} x(1)+B u(1) \\
= & \sum_{i=1}^{p} A_{i} x(1-i)+A_{0} \Psi(0, x(-p), \ldots, x(0)) \\
& +A_{0} G_{0} B u(0)+B u(1) \\
= & \Psi(1, x(-p), \ldots, x(0))+G_{1} B u(0)+G_{0} B u(1) .
\end{aligned}
$$

For $k \leq p$, we have

$x(k+1)$

$$
\begin{aligned}
= & \sum_{i=0}^{p} A_{i} x(k-i)+B u(k) \\
= & \sum_{i=k}^{p} A_{i} x(k-i)+\sum_{i=0}^{k-1} A_{i} x(k-i)+B u(k) \\
= & \sum_{i=k}^{p} A_{i} x(k-i) \\
& +\sum_{i=0}^{k-1} A_{i}(\Psi(k-i-1, x(-p), \ldots, x(0)) \\
& \left.\quad+\sum_{j=0}^{k-i-1} G_{k-i-1-j} B u(j)\right)+G_{0} B u(k)
\end{aligned}
$$

$$
\begin{aligned}
= & \left(\sum_{i=k}^{p} A_{i} x(k-i)\right. \\
& \left.+\sum_{i=0}^{k-1} A_{i} \Psi(k-i-1, x(-p), \ldots, x(0))\right) \\
& +\sum_{i=0}^{k-1} A_{i}\left(\sum_{j=0}^{k-i-1} G_{k-i-1-j} B u(j)\right)+G_{0} B u(k)
\end{aligned}
$$




$$
\begin{aligned}
= & \Psi(k, x(-p), \ldots, x(0))+\sum_{j=0}^{k-1} \sum_{i=0}^{k-j-1} A_{i} G_{k-i-1-j} B u(j) \\
& +G_{0} B u(k) \\
= & \Psi(k, x(-p), \ldots, x(0))+\sum_{j=0}^{k-1} G_{k-j} B u(j)+G_{0} B u(k) .
\end{aligned}
$$

For $k>p$, we have

$x(k+1)$

$$
\begin{aligned}
& =\sum_{i=0}^{p} A_{i} x(k-i)+B u(k) \\
& =\sum_{i=0}^{p} A_{i}(\Psi(k-i-1, x(-p), \ldots, x(0)) \\
& \left.+\sum_{j=0}^{k-i-1} G_{k-i-1-j} B u(j)\right)+G_{0} B u(k) \\
& =\left(\sum_{i=0}^{p} A_{i} \Psi(k-i-1, x(-p), \ldots, x(0))\right) \\
& +\sum_{i=0}^{p} A_{i}\left(\sum_{j=0}^{k-i-1} G_{k-i-1-j} B u(j)\right)+G_{0} B u(k) \\
& =\Psi(k, x(-p), \ldots, x(0)) \\
& +\sum_{j=k-p}^{k-1} \sum_{i=0}^{p-j} A_{i} G_{k-i-1-j} B u(j)+G_{0} B u(k) \\
& +\sum_{j=0}^{k-1-p} \sum_{i=0}^{p} A_{i} G_{k-i-1-j} B u(j)+G_{0} B u(k) \\
& =\Psi(k, x(-p), \ldots, x(0))+\sum_{j=0}^{k-1} G_{k-j} B u(j)+G_{0} B u(k) .
\end{aligned}
$$

\section{B. Proof of Lemma 4}

Proof. We introduce a new matrix sequence $\left\{W_{k}\right\}_{k=p+1}^{\infty} \in$ $\mathbb{R}^{n(p+1) \times n}$ given by

$$
W_{k}=\left[\begin{array}{c}
G_{k} \\
G_{k-1} \\
\vdots \\
G_{k-p}
\end{array}\right] .
$$

It is easy to verify that

$$
W_{k+1}=A W_{k}, \quad \forall k>p,
$$

where

$$
\mathbb{A}=\left[\begin{array}{cccc}
A_{0} & \cdots & A_{p-1} & A_{p} \\
I & \cdots & 0 & 0 \\
\vdots & \ddots & \vdots & \vdots \\
0 & \cdots & I & 0
\end{array}\right]
$$

By the well-known Hamilton-Caylay Theorem, for any $k \geq$ $n(p+1)+p$, we have

$$
W_{k} \in \operatorname{span}\left\{W_{p}, \ldots, W_{p+n(p+1)-1}\right\} .
$$

It follows that

$$
G_{k-p} \in \operatorname{span}\left\{G_{0}, \ldots, G_{n(p+1)-1}\right\} .
$$

Hence, we have

$$
\operatorname{span}\left\{G_{0}, \ldots, G_{k}, \ldots\right\}=\operatorname{span}\left\{G_{0}, \ldots, G_{n(p+1)-1}\right\} .
$$

\section{Proof of Lemma 9}

Proof. Consider the matrix sequence $\left\{W_{k}\right\}_{k=p+1}^{\infty} \in \mathbb{R}^{n(p+1) \times n}$ given by (B.1), it is easy to verify that

$$
H_{k}=W_{k}^{T}\left[\begin{array}{c}
B_{0} \\
\vdots \\
B_{p}
\end{array}\right], \quad \forall k \geq p .
$$

By the proof of Lemma 4, we have

$$
W_{k+1}=A W_{k}, \quad \forall k \geq p .
$$

This implies that

$$
\operatorname{span}\left\{H_{p}, \ldots, H_{k}, \ldots\right\}=\operatorname{span}\left\{H_{p}, \ldots, H_{n(p+1)+p-1}\right\} .
$$

Hence, we have

$$
\operatorname{span}\left\{H_{0}, \ldots, H_{k}, \ldots\right\}=\operatorname{span}\left\{H_{0}, \ldots, H_{n(p+1)+p-1}\right\} .
$$

\section{Acknowledgments}

The authors would like to thank the Associate Editor, Professor Valery Y. Glizer, and the anonymous reviewer for their valuable comments and suggestions, which significantly contributed to improving the quality of the publication. This work is supported by the National Natural Science Foundation (NNSF) of China (60774089, 10972003), the Foundation Grant of Guangxi Key Laboratory of Automobile Components and Vehicle Technology (13-A-03-01), and the Opening Project of Guangxi Key Laboratory of Automobile Components and Vehicle Technology (2012KFZD03). It is also supported by the Beijing City Board of Education Science and Technology Program: modeling, analysis, and control of swarming behavior of multiple dynamic agent systems. 


\section{References}

[1] R. E. Kalman, "On the general theory of control systems," in Proceedings of the 1st IFAC Congress, vol. 1, pp. 481-492, Moscow, Russia, 1960.

[2] T. Kailath, Linear Systems, Prentice-Hall, Englewood Cliffs, NJ, USA, 1980.

[3] E. D. Sontag, Mathematical Control Theory: Deterministic Finite Dimensional Systems, vol. 6, Springer, New York, NY, USA, 1990.

[4] H. W. Sorenson, "Controllability and observability of linear, stochastic, time-discrete control systems," in Advances in Control Systems, vol. 6, pp. 95-158, Academic Press, New York, NY, USA, 1968.

[5] J. Klamka, "Controllability of delayed dynamical systems," in Proceedings of 14th IFAC congress, vol. 4, pp. 485-490.

[6] M. Fliess, "Some new interpretations of controllability and their practical implications," Annual Reviews in Control, vol. 23, pp. 197-206, 1999.

[7] J. Klamka, "Relative and absolute controllability of discrete systems with delays in control," International Journal of Control, vol. 26, no. 1, pp. 65-74, 1977.

[8] K. Watanabe, "Further study of spectral controllability of systems with multiple commensurate delays in state variables," International Journal of Control, vol. 39, no. 3, pp. 497-505, 1984.

[9] V. N. Phat, "Controllability of discrete-time systems with multiple delays on controls and states," International Journal of Control, vol. 49, no. 5, pp. 1645-1654, 1989.

[10] V. Y. Glizer, "Euclidean space controllability of singularly perturbed linear systems with state delay," Systems \& Control Letters, vol. 43, no. 3, pp. 181-191, 2001.

[11] J. Klamka, "Absolute controllability of linear systems with timevariable delays in control," International Journal of Control, vol. 26, no. 1, pp. 57-63, 1977.

[12] H. Shi, G. Xie, and W. Luo, "Controllability analysis of linear discrete-time systems with time-delay in state," Abstract and Applied Analysis, vol. 2012, Article ID 490903, 11 pages, 2012. 


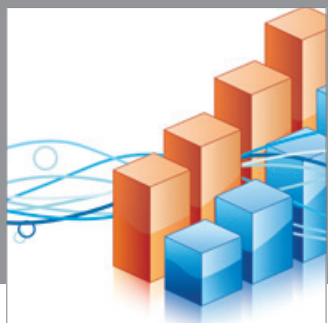

Advances in

Operations Research

mansans

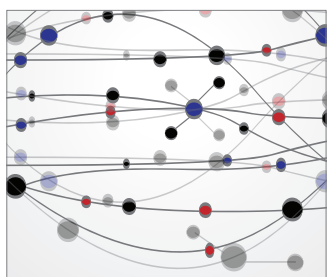

The Scientific World Journal
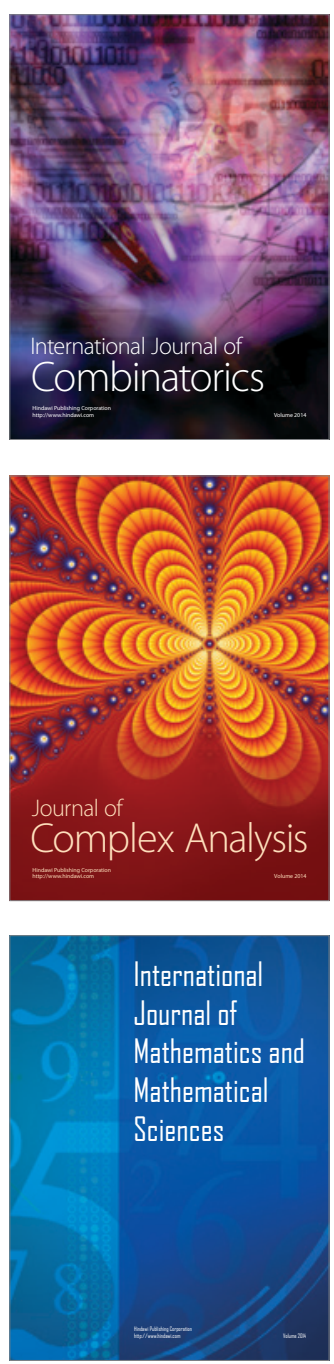
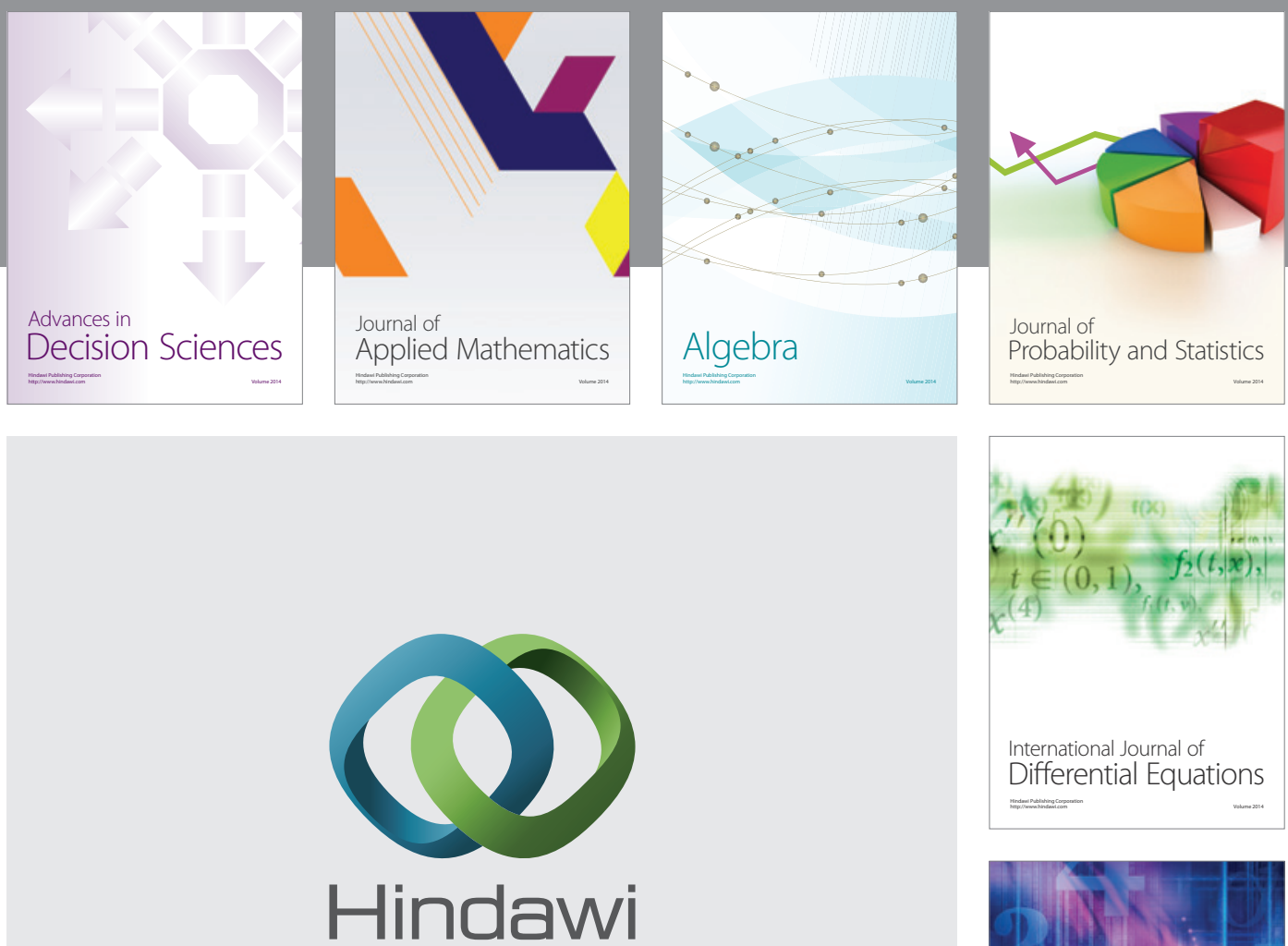

Submit your manuscripts at http://www.hindawi.com
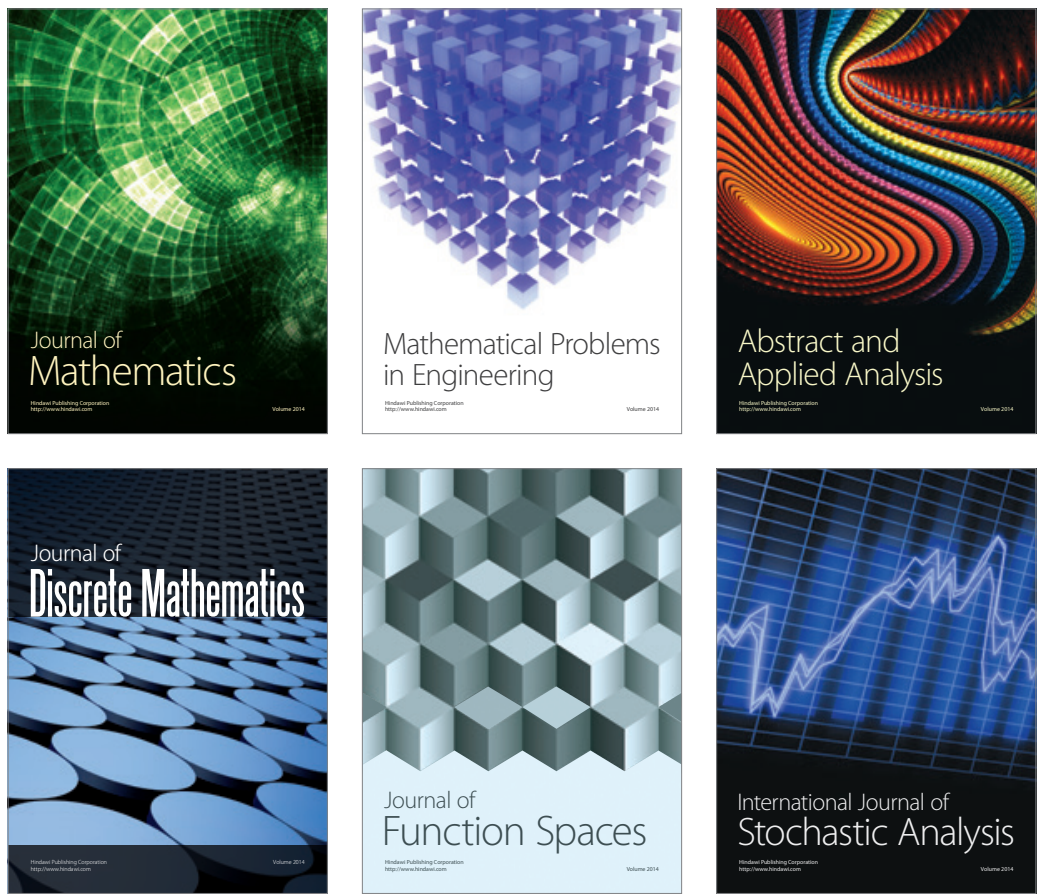

Journal of

Function Spaces

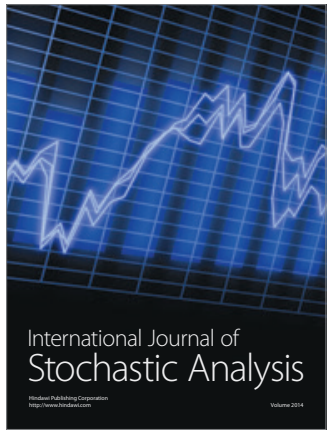

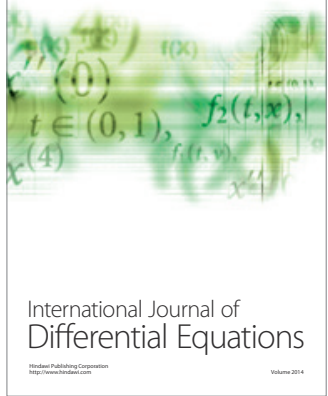
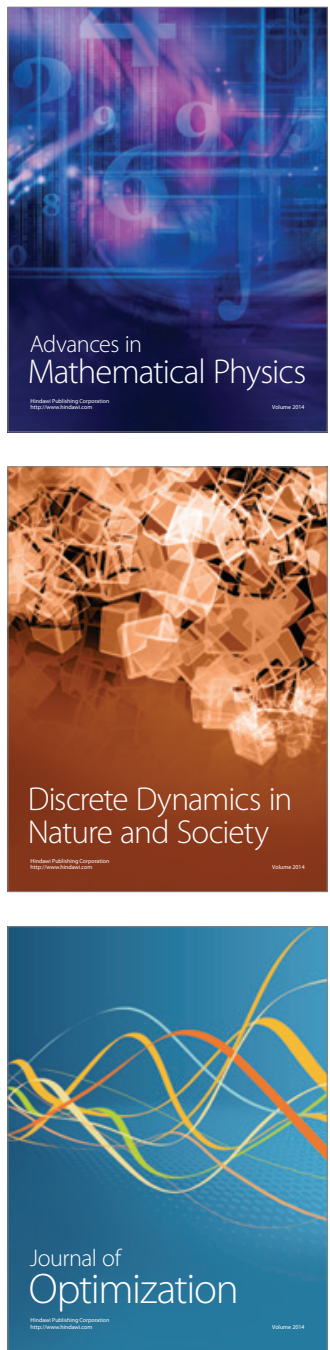